\title{
Development of the Department of Medicine for the Elderly Liaison Team
}

\author{
Authors: Elinor Burn, ${ }^{\mathrm{A}}$ Barry Evans, ${ }^{\mathrm{A}}$ Adam Gordon, ${ }^{\mathrm{A}, \mathrm{B}}$ Tanuja Shah ${ }^{\mathrm{A}}$ and Frances Grant ${ }^{\mathrm{A}}$
}

\section{Aims}

To improve the care of patients with complexity, multimorbidity and frailty within the acute hospital, through the development of a specialist liaison team.

\section{Methods}

Older people undergoing emergency surgery encounter more postoperative medical problems. The National Emergency Laparotomy Audit (NELA) includes the process measure that patients over 70 should have access to specialists in the care of older patients. This led to the collaboration between critical care and surgical teams and development of the liaison service $-a$ consultant geriatrician, specialist registrar and nurse consultant. This began an integrated way of working to improve care for emergency laparotomy patients over 70 .

Due to the success of the service, it has expanded to incorporate twice-weekly board rounds on urology and subsequently general surgery, to highlight patients who would benefit from geriatrician input. This service acts to incorporate the wider multidisciplinary team (MDT) to create structured management and coordinated discharge planning.

To ensure development of this service, year-long fellowship posts have been created. These registrars deliver the clinical responsibilities with focus on quality and service improvement, while developing leadership skills through a formal qualification.

The liaison team is also responsible for the Department of Medicine for the Elderly referrals across the wider hospital. This highlighted the referral system, which required improved communication and documentation of advice. We now operate an electronic referral system maintaining our active patient list with a concise review sticker to improve this communication, with a recognisable logo for audit record-keeping.

\section{Results}

Using NELA criteria, the percentage of patients seen by a geriatrician post-laparotomy increased from $7 \%$ to $93 \%$ over 2 years. Statistical process control (SPC) (Fig 1) demonstrates that introduction of the service was associated with reduction in mean length of stay (LOS) of 3 days per admission ( 22 to 19 days), with reduced variability.

Authors: A Department of Medicine for the Elderly, Royal Derby Hospital; ${ }^{B}$ Division of Medical Sciences and Graduate Entry Medicine, University of Nottingham, Derby, UK

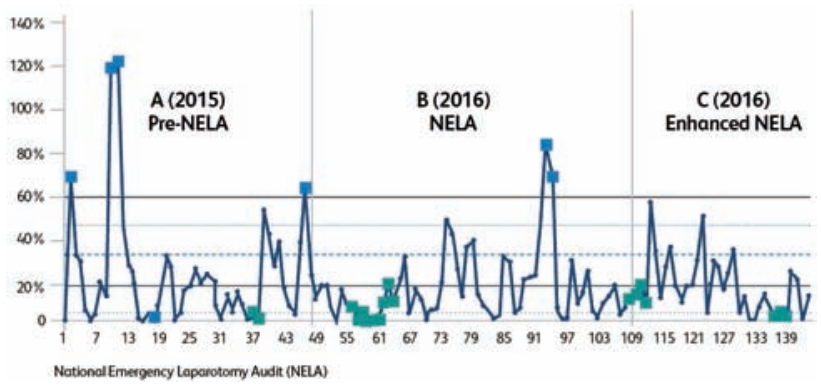

Fig 1. Statistical process control chart of LOS related to NELA service intervention

NHS Improvement figures of $£ 400 /$ bed day and trust activity figures indicating 100 emergency laparotomies in the over 70s/year equates to a cost-saving of $£ 120,000 /$ year.

Reviewing urology board rounds through an SPC chart demonstrated a mean reduction of 1.68 days (11.34 to 9.66 days). This stemmed from a reduction in LOS exceeding the third standard deviation.

Qualitative survey data collected from surgeons, anaesthetists and ward-based MDTs demonstrated unanimous satisfaction with the service and how it supported staff to deliver better patient care with more timely resolution of medical and discharge issues through more prompt MDT discussions.

\section{Conclusion}

Management of older patients with complexity is everyone's business. Surgical, critical care and liaison teams have worked collaboratively to realise integrated care for older people. Case finding through twice-weekly geriatric medicine and surgical team MDTs has enabled more timely recognition of older people with frailty; specialist support enables rapid resolution in problems with reduction in length of stay and cost savings to the trust. Clear documentation and communication of advice has improved understanding of the complexities and outstanding problems of these patients. The model is simple, cost-effective and could be translated and operationalised to other NHS organisations.

\section{Conflict of interest statement}

None to declare. 